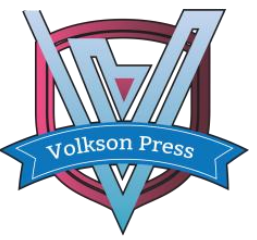

Contents List available at VOLKSON PRESS

Mechanical and Control Engineering (MCE)

DOI : http://doi.org/10.26480/wsmce.01.2017.118.120

\title{
A NEW METHOD OF CORRELATION REGION LEAST SQUARE ALGORITHM FOR EARTH SYSTEM PARAMETER IDENTIFICATION
}

\author{
Sun Guoliang ${ }^{1}$, Wang Shuo ${ }^{1}$, Qin Honglei ${ }^{1}$, Cao Weihua ${ }^{1,2^{*}}$ \\ ${ }^{1}$ School of Electronics Information Engineering, Beihang University, No.37th, Xueyuan Rd, Beijing, China. \\ ${ }^{2}$ College of Mechanical and Control Engineering, Guilin University of Technology, No.12th, Jiangan Rd, Guilin, China. \\ *Corresponding Author Email: caoweihuaxixi@126.com
}

This is an open access article distributed under the Creative Commons Attribution License, which permits unrestricted use, distribution, and reproduction in any medium, provided the original work is properly cited

\section{ARTICLE DETAILS}

\section{Article History:}

Received 02 october 2017 Accepted 06 october 2017 Available online 11 november 2017

\section{Keywords}

Least square, pseudo random msequence, correlation, parameter identification, earth system

\section{ABSTRACT}

In the earth exploration field, least square algorithm is one of the most commonly traditional methods for earth parameter system identification. Aiming at the problems of the traditional least square algorithm based on the input and output signal that the earth system is susceptible to noise and not so accurate identification, in this it has proposed a new method of correlation region least square algorithm for the earth system parameter identification. The new method proposes that input $m$-sequence to the unknown earth system, then using the characteristics of the noise can be suppressed by using the auto correlation of the system input signal and the cross correlation of the inputoutput signal, and using those signals of correlation region to carry out least square calculation, so that the unknown parameters of the earth system are obtained. The computer simulation of earth system identification has showed that the new method got great improvement than the traditional ones in suppressing noise interference and improvement identification precision.

\section{Introduction}

In the earth system exploration, researchers can establish a mathematical model for approximate earth system based on understanding of the earth or prior knowledge, then it can recognize earth system through identification the mathematical model parameters [1]. In particularly, least square method is widely used to recognize the earth system parameters. Traditional method of earth system parameter identification is that input pseudo-random $m$ sequence signal to system, and collecting output signal of the system [2-4]. Then using the input and output signal for the least square algorithm to obtain unknown parameters, so it can recognize earth system. However, the production practice shows the traditional method that is susceptible to noise and not so accurate to identification earth system parameters [5-7].

With the increasing request of identification accuracy in modern production technology, and the limitations of traditional least square method for system parameter identification, so a number of improved least square methods have been derived. Such as recursive least squares algorithm, fading memory recursive least-squares, these least square methods are contributing to improve data processing in some degree $[8,9]$. However, those methods are still susceptible to system noise and deviation from the actual system parameter. To improve accurate for identification earth system parameter, a new method was put forward by using least square algorithm based on the correlation of system input and output pseudo random m-sequence signal.

\section{METHODS AND MATERIALS}

\subsection{Earth system parameter model}

In engineering practice, it shows earth system with linear invariant features, so a linear mathematical model can be used to describe the earth system [8]. Therefore, the earth system parameters model can be presented as Eq. (1).

$G(z)=\frac{y(k)}{u(k)}=\frac{b_{1} z^{-1}+b_{2} z^{-2}+\ldots+b_{n} z^{-n}}{1+a_{1} z^{-1}+a_{2} z^{-2}+\ldots+a_{n} z^{-n}}$

Where $a_{i}$ and $b_{i}$ are earth system unknown parameters, $u(k)$ is the $\mathrm{k}$-th $\mathrm{m}$-sequence signal input the system,

$y(k)$ is the system output signal base on $u(k)$,

If there is noise $v(k)$ in the system, the Eq. (1) can be expressed as Eq. (2):

$A\left(z^{-1}\right) y(k)=B\left(z^{-1}\right) u(k)+v(k)$

Where $A\left(z^{-1}\right)=1+a_{1} z^{-1}+\ldots+a_{n} z^{-n}, B\left(z^{-1}\right)=1+b_{1} z^{-1}+\ldots+b_{n} z^{-n}$.

Based on the mathematical model of the earth parameters system, then using a method to obtain the unknown parameters, so the earth system can be identified.

\subsection{Traditional Least Square Method}

Using the traditional least square method of earth system identification to find the unknown parameters $a_{i}$ and $b_{i}$ from Eq. (1), the coefficient can be defined as matrix $\theta$ to be estimated:

$\theta=\left[a_{1}, a_{2}, \ldots, a_{n}, b_{1}, b_{2}, \ldots, b_{n}\right]^{T}$

Meanwhile, the system input signal $u(k)$ and output signal $y(k)$ is defined as the matrix $h(k)$ :

$\mathrm{h}(\mathrm{k})=[-\mathrm{y}(\mathrm{k}-1),-\mathrm{y}(\mathrm{k}-2), \ldots \ldots . \mathrm{y}(\mathrm{k}-\mathrm{n}), \mathrm{u}(\mathrm{k}-1), \mathrm{u}(\mathrm{k}-2) \ldots \ldots \mathrm{u}(\mathrm{k}-\mathrm{n})]$

The system output included noise $v(k)$ can be expressed as Eq.(5) :

$$
z(k)=h(k) \theta+v(k)
$$

To find the estimated $\hat{\theta}$ of $\theta$, minimizing the square sum of the differences between each measurement date $z_{m}(\mathrm{~m}=1,2, \ldots \mathrm{n})$ and the estimate $\hat{z}_{m}=H_{m} \hat{\theta}$, constructing a guideline function and minimizing it as Eq. (6): 
$J(\hat{\theta})=\left(Z_{m}-H_{m} \hat{\theta}\right)^{T}\left(Z_{m}-H_{m} \hat{\theta}\right)=\min$

Define the guideline function Eq. (6) as a partial derivative of $\theta$, and make it for 0 , then find the extreme value.

$\left.\frac{\partial J}{\partial \theta}\right|_{\theta=\hat{\theta}}=-2 H_{m}^{T}\left(Z_{m}-H_{m} \hat{\theta}\right)=0$

Therefore, $H_{\mathrm{m}}^{T} H_{\mathrm{m}} \hat{\theta}=H_{\mathrm{m}}^{T} Z_{\mathrm{m}}$.

By the procedure mentioned above, the unknown parameters $a_{i}$ and $b_{i}$ can be obtained, and it can achieve identification of earth system parameters. However, the traditional method directly take advantage of the system's input and output signal for least square calculation, only in rare cases where the output noiseless or error is zero-mean of Gaussian white noise, least square estimate is equivalent to the solution of the maximum likelihood estimation [10]. For the system input signal $x(k)$ and output signal $y(k)$ are exist errors or disturbances, the least square estimation of the traditional method will no longer is the best, but there are deviation estimates. There are defects that are low precision for earth system identification. So it requires a better way to recognize system parameters by treating the noise as zero or zero mean.

\subsection{Correlation Region Least Square Algorithm}

This paper addresses the shortcomings of the traditional least square algorithm, in order to improve the identification accuracy of the earth system parameters, it has proposed a new method based on the correlation domain least squares algorithm [11]. The new method is as follows:

According to the earth system has the characteristics of linear timeinvariant, establishing a linear mathematical model for the earth system as equation (1). Then the $\mathrm{m}$ - sequence signal of T period input to the earth system, the system output signal $y(t)$. And the autocorrelation function $R_{x x}(\tau)$ of the input signal $x(t)$ is calculated as follow:

$R_{x x}(\tau)=\frac{1}{T} \int_{0}^{T} x(t) x(t+\tau) d t$

Seeking the cross-correlation function $R_{x y}(\tau)$ from system input signal $x(t)$ and output signal $y(t)$ as follow:

$R_{x y}(\tau)=\frac{1}{T_{0}} \int_{0}^{T_{0}} x(t) y(t+\tau) d t$

Without changing the system's input signal, the system autocorrelation of input signal is the least squares input signal, and the cross-correlation of system input -output signal is the least-squares output signal. Ie, the variables for least squares calculation are as follows.

Input: $u(t)=R_{x x}(t)$

Output: $z(t)=R_{x y}(t)$

Using the above input $\mathrm{u}(\mathrm{t})$ and output $\mathrm{z}(\mathrm{t})$ data are defined as a system matrix:

$h(k)=\left[-R_{x y}(k-1),-R_{x y}(k-2), \ldots-R_{x y}(k-n), R_{x x}(k-1), R_{x x}(k-2) \ldots \ldots R_{x x}(k-n)\right]$

The system model coefficient is defined as the matrix to be estimated:

$\theta=\left[a_{1}, a_{2}, \ldots, a_{n}, b_{1}, b_{2}, \ldots, b_{n}\right]^{T}$

The above operations take advantage of the dependence of the $\mathrm{m}$ sequence on the suppression of system noise, so the system input and output signal are both corrupted by noise can be expressed as follow: $v(k)$ is noise with a zero mean, $\theta$ is parameter matrix to be estimated, to find $\hat{\theta}$ that is the estimated value of $\theta$, minimizing the square sum of the differences between each measurement value $R_{x y \mathrm{i}}(k)(\mathrm{i}=1,2, \ldots . . ., \mathrm{n})$ and the estimated $\hat{R_{x y \mathrm{i}}}=H_{\mathrm{i}} \hat{\theta}$. So guidelines can be constructed, which minimized the sum of the squares of the differences between the crosscorrelation measurements and the calculated estimates:

$$
J(\hat{\theta})=\left(R_{x y \mathrm{~m}}-H_{\mathrm{m}} \hat{\theta}\right)^{T}\left(R_{x y \mathrm{~m}}-H_{\mathrm{m}} \hat{\theta}\right)=\min
$$

Deriving the above guideline function for partial derivative of $\theta$ and make it for 0 , find the extreme value:

$\left.\frac{\partial J}{\partial \theta}\right|_{\theta=\hat{\theta}}=-2 H_{\mathrm{m}}^{T}\left(R_{x y \mathrm{~m}}-H_{\mathrm{m}} \hat{\theta}\right)=0$

So got the following correspondence:

$$
H_{\mathrm{m}}^{T} H_{\mathrm{m}} \hat{\theta}=H_{\mathrm{m}}^{T} R_{x y \mathrm{~m}}
$$

If $H_{m}$ the number of rows is greater than or equal to the number of columns, 即 $m \geq 2 n, H_{m}^{T} H_{m}$ is full-rank, $\left(H_{m}^{T} H_{m}\right)^{-1}$ is existence, then the $\theta$ estimated value is $\hat{\theta}=\left(H_{\mathrm{m}}{ }^{T} H_{\mathrm{m}}\right)^{-1} H_{\mathrm{m}}{ }^{T} R_{x \mathrm{~m}}$.

The unknown parameters $a_{i}$ and $b_{i}$ can be obtained, and the result is approximating the actual characteristics of the earth system.

Based on above study, the least squares algorithm using correlation of $\mathrm{m}$ sequence, its advantage is in use of the autocorrelation of system input $m$ sequence signal and the cross-correlation input-output signal. Those can take average of system noise for zero. And it can suppress the system noise interference and improve the system parameter identification accuracy.

\section{SIMULATION ANALYSIS}

In order to compare the application effect of reducing noise between traditional least square method and correlation region least square algorithm in the earth system identification, using MATLAB software to test and simulation analysis.

Due to the earth system with linear invariant features, and the RCC2 circuit model has characteristics of linear time invariant. Therefore, RCC2 circuit model is often used as a simulation model of the earth system. RCC2 structure is shown as figure 1 , witch parameters are configured as follows : $R_{0}=350 \Omega, R_{1}=50 \Omega, R_{2}=100 \Omega, C_{1}=0.01 F, C_{2}=10 \mu F$. The m-sequence signal input RCC2 circuit system, and the initial parameters of the $\mathrm{m}$-sequence signal are as follows: the order is 11 , the sampling period is 0.002 second, the oversampling factor is 10 , and the amplitude is 1 .

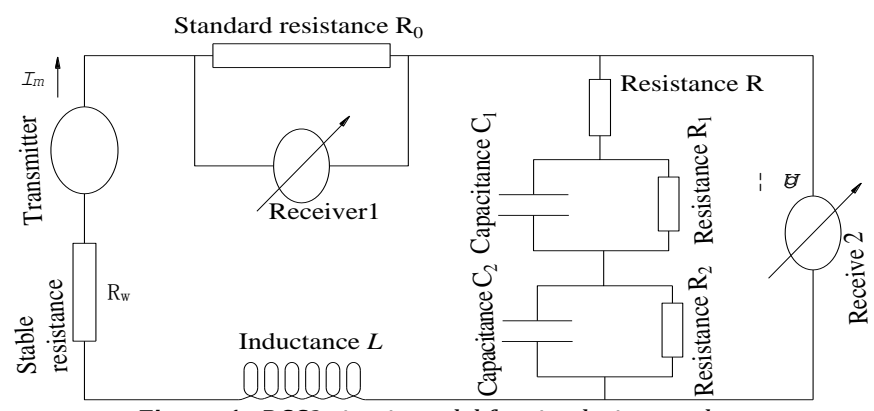

Figure 1: RCC2 circuit model for simulating earth system

In different noise conditions, the system parameter identification accuracy is compared between correlation region least-squares algorithm and traditional least-squares algorithm. The figure 2, figure 3 and figure 4 respectively represent system step response curve when SNR is $30 \mathrm{db}$, $20 \mathrm{db}$ and $10 \mathrm{db}$.

$$
R_{x y}(k)=h(k) \theta+v(k)
$$




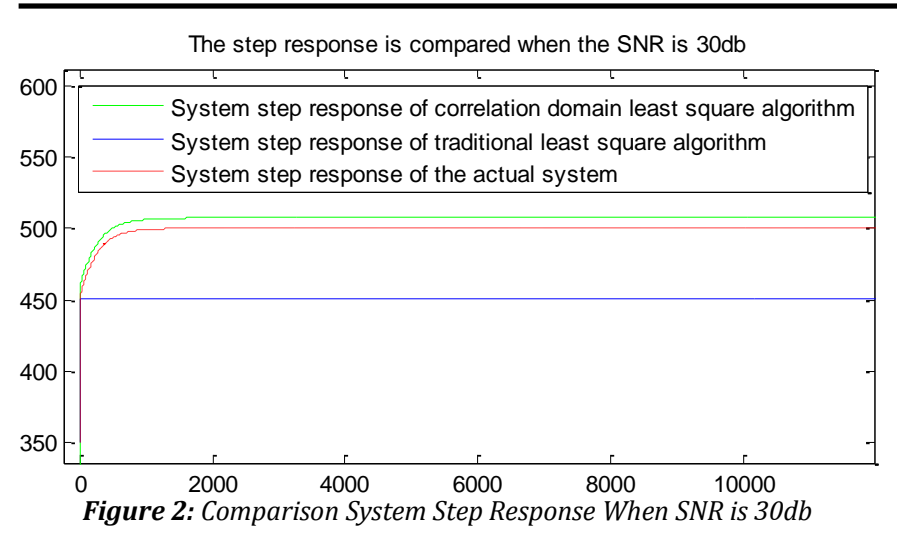

The step response is compared when the SNR is $20 \mathrm{db}$

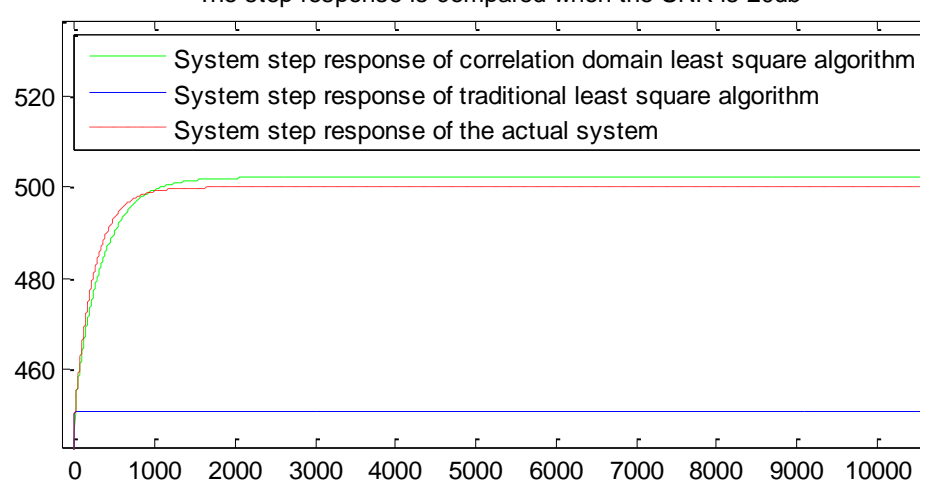

Figure 3: Comparison System Step Response When SNR is 20db

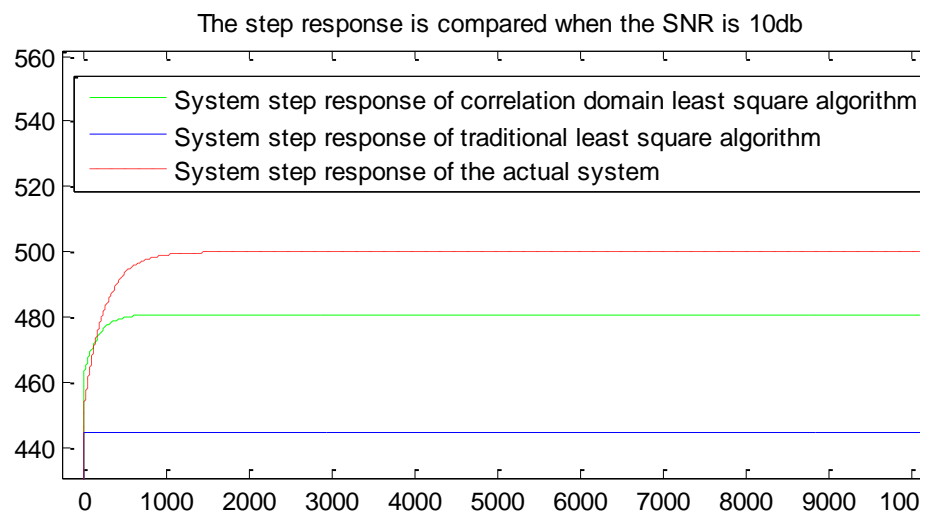

Figure 4: Comparison System Step Response When SNR is $10 \mathrm{db}$

According to the figure 2, figure 3 and figure 4 in different noise conditions, analyzed the deference of system step response curve between traditional least square algorithm and correlation region least square algorithm. The results have showed system step response curve based on correlation region least square algorithm that is closer to the actual system, and which the error is smaller. The recognition accuracy is improved relatively traditional method. The reason is that the new method exploits correlation properties of $\mathrm{m}$-sequences to effectively suppress noise, but the traditional least square algorithm is directly using the system's input and output signal to identification system parameters, it was not processed noise, so the recognition accuracy is in poor [12]

\section{CONCLUSION AND DISCUSSION}

In this paper, a new method of the earth system identification based on the correlation domain least squares algorithm is proposed. Specifically, input the m-sequence to unknown system, and collect output signal, the data for least-squares algorithm is from the autocorrelation of the input signal and the cross correlation of the input-output signal. Therefore, the unknown parameters of the earth system can be obtained. Finally, comparing with the recognition effect of the new method and traditional method in different noise conditions through computer simulation, it showed the new method that can effectively suppress noise of earth system and improve the identification accuracy of system parameters.

\section{REFERENCES}

[1] Shuang, L. 2005. Research on models and algorithms of geodesy and geophysics joint inversion [D]. Wuhan University, Wuhan.

[2] Xianzhi, C., Chong, C. 2005.N Parameter identification of time -varying systems based on the least-squares modified algorithm. Journal of Fuzhou University (Natural Sciences Edtion), 2 (33), 163-166.

[3] Yong, Z., Hui, Y., Feng, D. 2006. System identification method for colored noise. Journal of Nanjing University of Aeronautics and Astronautics, 38 (2), 167-171. DOI : 10.16356/j.1005-2615. 2006.s1.042

[4] Shaoheng, C., Rujun, C., Minghui, G. 2014. Review of the pseudorandom $\mathrm{m}$ sequence and its application in electrical prospecting of exploration Geophysics. Progress in Geophysics, 29 (01), 439-446.

[5] Jingwan, L. 2004. Application of minimum diplo-multiply method in system identification. Journal of Beijing Institute of Civil Eng and Architecture , 12 (3),19-22

[6] Sun, J.C., Zhou, Y.T., Bai, Y.H · 2008. Nonlinear noise reduction of chaotic time series based on multidimensional recurrent least squares support vector machines · Neu-ro computing,71 (16), 3675-3679.

[7] Jinfeng, H., Hexin, Z. 2011. A modified method of correlated identification based on pseudo random m-sequence signal. Electronics Optics and Control, 18 (7),13-18.

[8] Junhong, C. 2012. Least square method and its application in the inversion problem of earth deformation. Journal of Xi An University of Arts and Science (Nat Sci Ed),15 (04), 68-70.

[9] Siqing, L.2013. Application of least square principle surveying engineering. Mine Surveying, 11 (1), 97-101.

[10] Dongsheng, S., Peiqiang, Z., Yaowu, W. 2013. Simulation of parameter identification for gun control system based on RLS. Journal of System Simulation , 25 (8), 1726-1729.

[11] Shuo, W. 2015. Research on the method of geodetic electric measurement based on pseudo random sequence [D]. Beihang University, Beijing.

[12] Jiande, H. 2014. Mathematical models in electromagnetic survey. Mathematics in Practice and Theory, 3 (2), 27-31. 